\title{
Mode of Inoculation of the Lyme Disease Agent Borrelia burgdorferi Influences Infection and Immune Responses in Inbred Strains of Mice
}

\author{
Lise Gern, Ulrich E. Schaible, and Markus M. Simon
}

\author{
Institut de Zoologie. Université de Neuchâtel. Switzerland: \\ Max-Planck-Institut fir Immunbiologie, Freiburg, Germany.
}

\begin{abstract}
Mice were infected with Borrelia burgdorferi by infection via Ixodes ricinus and experimental inoculation to determine whether transmission rates of spirochetes and antibody responses are influenced. Mice infected by the natural route were substantially more infective for ticks; two- to sixfold more tick larvae were positive for $\boldsymbol{B}$. burgdorferi than those fed on experimentally inoculated mice. In natural infection, spirochetemia may be greater or spirochetes may be more accessible for transmission. Thus, this form of xenodiagnosis could be used to determine levels of spirochetes in the vertebrate host. Similar levels of antibody were present in all mice; however, those infected by the natural route lacked antibodies to outer surface proteins (Osp) A and B. The small antigen dose given through a tick bite may not have been sufficient to induce rapid OspA or OspB antibodies, thereby allowing the later development of higher levels of spirochetemia.
\end{abstract}

Since the discovery of Borrelia burgdorferi as the cause of Lyme borreliosis, most information on the pathogenesis of the disease and the immune responses to $B$. burgdorferi derives from laboratory models in which animals were experimentally inoculated with viable spirochetes. In mice, hamsters, and white-footed mice (Peromyscus leucopus), the first antibodies detected were those to the outer surface proteins (Osp) A and B, to a 39-kDa protein, and to the 41-kDa flagellin (fla) [1-3]. It was subsequently shown that antibodies specific for OspA and OspB are protective and able to prevent $B$. burgdorferi-induced disease in immunodeficient SCID $[4,5]$ and immunocompetent $\mathrm{C} 3 \mathrm{H} / \mathrm{HeJ}$ mice $[6,7]$.

In contrast to results in experimentally inoculated mice, the first antibodies to be detected in patients with Lyme borreliosis recognize the $41-\mathrm{kDa}$ flagellin or the $39-\mathrm{kDa}$ antigen (or both) but not OspA or OspB [8]. The differential immune responses of experimentally inoculated mice versus patients with Lyme disease may be related to the mode of inoculation of spirochetes, as suggested by a study in dogs [9]; only experimentally but not naturally infected dogs expressed antibodies to OspA.

The present study was undertaken to investigate the influence of the route of inoculation of $B$. burgdorferi on infection and antibody response in 3 inbred strains of mice.

\section{Materials and Methods}

B. burgdorferi. The European strain ZS7 used in this study was cultivated and quantified as described previously [10].

\footnotetext{
Received I May 1992; revised 30 October 1992.

Grant support: Swiss National Science Foundation (32-29964.90) and Bundesministerium für Forschung und Technologie (K| 8909/8).

Reprints or correspondence: Dr. Lise Gern, Institut de Zoologie. Chantemerle 22, CH-2000 Neuchâtel. Switzerland.
}

The Journal of Infectious Diseases 1993;167:971-5 (C) 1993 by The University of Chicago. All rights reserved 0022-1899/93/6704-0029\$01.00
Recombinant OspA. Recombinant OspA from strain ZS7 was expressed from the puEXI expression vector in Escherichia coli. The protein was extracted and purified by affinity chromatography using an OspA-specific monoclonal antibody as described previously [5].

Tick colony. The larval and nymphal $I$. ricinus ticks used in this study were derived from a $B$. burgdorferi-free laboratory colony maintained at the Institute of Zoology (Neuchâtel).

Animals. Adult mice of strains AKR/N $\left(\mathrm{H}-2^{\mathrm{k}}\right), \mathrm{C} 3 \mathrm{H} / \mathrm{HeJ}$ $\left(\mathrm{H}-2^{\mathrm{k}}\right)$, and DBA/2 $\left(\mathrm{H}-2^{\mathrm{d}}\right)$ were bred at the Institute of Zoology. Females 8-10 weeks old were used.

Infection procedures. Unfed I. ricinus nymphs were infected using the modified capillary method [11]. Strain ZS7 at a concentration of $1 \times 10^{8}$ cells $/ \mathrm{mL}$ BSK II medium was used to infect the ticks. Ticks took up $\sim 1.5 \mu \mathrm{L}\left(\sim 1.5 \times 10^{5}\right.$ spirochetes). Each experimental group of mice included $2 \mathrm{AKR} / \mathrm{N}, 2$ $\mathrm{DBA} / 2$, and $2 \mathrm{C} 3 \mathrm{H} / \mathrm{HeJ}$ mice.

For group I, 6 mice were inoculated subcutaneously (sc) in the tail with $0.2 \mathrm{~mL}$ of BSK II medium containing $2 \times 10^{7} \mathrm{~B}$. burgdorferi ZS7 organisms.

For group II, 6 mice were inoculated with a suspension of infected $I$. ricinus nymphs. Immediately after the infection by capillary, 42 infected nymphs were triturated in $1.5 \mathrm{~mL}$ of BSK II medium. Spirochetes were counted and adjusted to $3 \times 10^{6}$ spirochetes $/ \mathrm{mL} ; 0.2 \mathrm{~mL}$ of the suspension $\left(\sim 6 \times 10^{5}\right.$ spirochetes/mouse) was inoculated sc in the tail of each mouse.

For group III, 6 mice were exposed to 7 artificially infected $I$. ricinus nymphs. Nymphs were fed in two hollow plastic caps fixed bilaterally on the back of the mouse, and a collar was placed around the neck to prevent grooming. Engorged ticks (4-6) were collected from each mouse.

Xenodiagnosis and spirochete detection in ticks. To determine the level of spirochetemia, infected mice were exposed to uninfected I. ricinus larvae at 14 (xenodiagnosis I, 80 larvae/ mouse) and 100 days (xenodiagnosis II, 60 larvae/mouse) after infection. Larvae were put on the head of the mouse, and a collar was placed around the neck to prevent grooming. Mice were kept in separate cages over trays of water, and engorged ticks that had dropped into water were collected daily, placed into vials, and stored at room temperature and $95 \%$ humidity. 
Ticks were evaluated for $B$. burgdorferi after molting using a direct immunofluorescence antibody test [12].

Western blot and ELISA. Sera derived from infected mice were analyzed by Western blots of spirochetal lysates (strain ZS7) and by ELISA with either soluble B. burgdorferi (ZS7) antigen or recombinant $\mathrm{OspA}$ as described [5].

\section{Results}

Inbred mice of strains AKR/N (H-2 $), \mathrm{C} 3 \mathrm{H} / \mathrm{HeJ}\left(\mathrm{H}-2^{k}\right)$, and DBA/2 (H-2d) were infected with $B$. burgdorferi by experimental inoculation with in vitro-propagated spirochetes (group I), experimental inoculation with a tissue suspension of infected ticks (group II), and natural infection via tick bites (group III). The numbers of spirochetes transferred to mice via syringe were $\sim 2 \times 10^{7}$ for group I and $\sim 6 \times 10^{5}$ for group II. The numbers of spirochetes transmitted via tick bites can only be estimated and were $\sim 1 \times 10^{6}$ at most.

At 14 and 100 days after infection, mice were exposed to uninfected $I$. ricinus larvae to asses their capacity to transmit spirochetes. Mice from each of the 3 inbred strains previously infected via tick bites (group III) were shown to infect, on average, about two- to fourfold more $I$. ricinus larvae (42.5\% at day 14 and $88 \%$ at day 100 ) than mice infected via syringe with either $B$. burgdorferi organisms (group I, $6 \%$ on day 14 and $37.5 \%$ at day 100 ) or a tissue suspension of infected ticks (group II, $10 \%$ at day 14 and $33.3 \%$ at day 100 ). In all 3 strains of mice, infection rates of $I$. ricinus larvae were significantly increased (two- to sixfold) at day 100 compared with those at day 14 independent of the inoculation protocol, reaching $88 \%$ (twofold increase) after tick infection (group III) and between $37.5 \%$ (sixfold increase, group I) and $33.3 \%$ (threefold increase, group II) after experimental inoculation.

Serum samples were taken from mice at days $7,14,26,40$, 63,77 , and 110 after infection and analyzed individually for $B$. burgdorferi-specific antibodies by ELISA. All mice, independent of strain or inoculation protocol, expressed similar amounts of specific antibodies as assessed on total cell lysates of B. burgdorferi strain ZS7 (figure 1A). However, when assayed on purified recombinant OspA [5] alone, specific antibodies were found only in experimentally inoculated (group I, II) but not in tick-infected mice (group III, figure 1B).

To verify these results, individual sera were analyzed for antibody specificities by Western blot. Figure 2 depicts representative banding patterns obtained with sera from $A K R / N$, $\mathrm{C} 3 \mathrm{H} / \mathrm{HeJ}$, and DBA/2 mice at days $7,14,26$, and 40 after inoculation. Sera from mice experimentally inoculated with either spirochetes (group I) or lysates derived from infected ticks (group II) contained antibodies to OspA (3I kDa) and OspB ( $34 \mathrm{kDa}$ ) in addition to various other $B$. burgdorferi structures, including flagellin (41-kDa) and antigens with approximate molecular masses of $14,18,22,26,39,65$, and $70 \mathrm{kDa}$. In contrast, sera from mice inoculated via infected

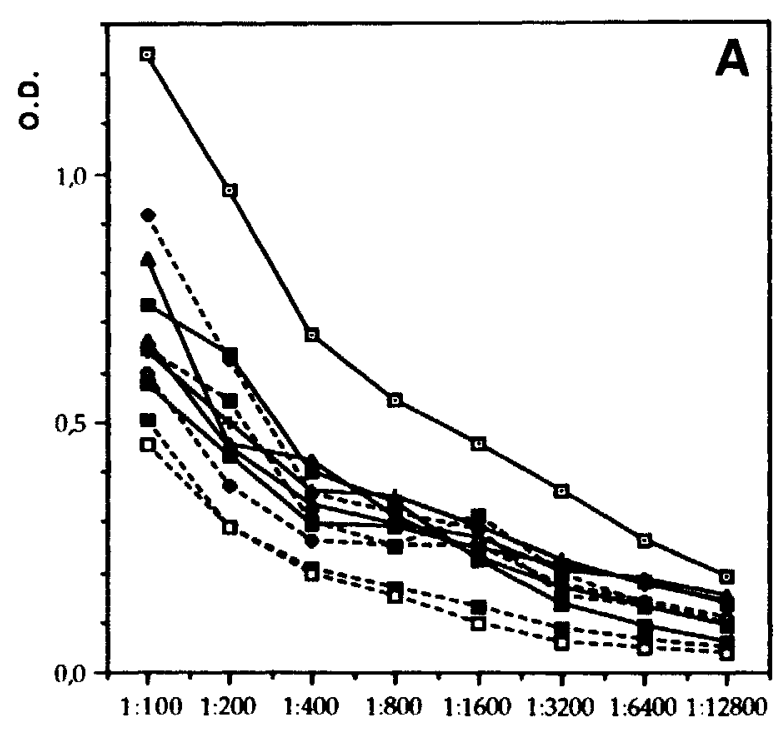

dil

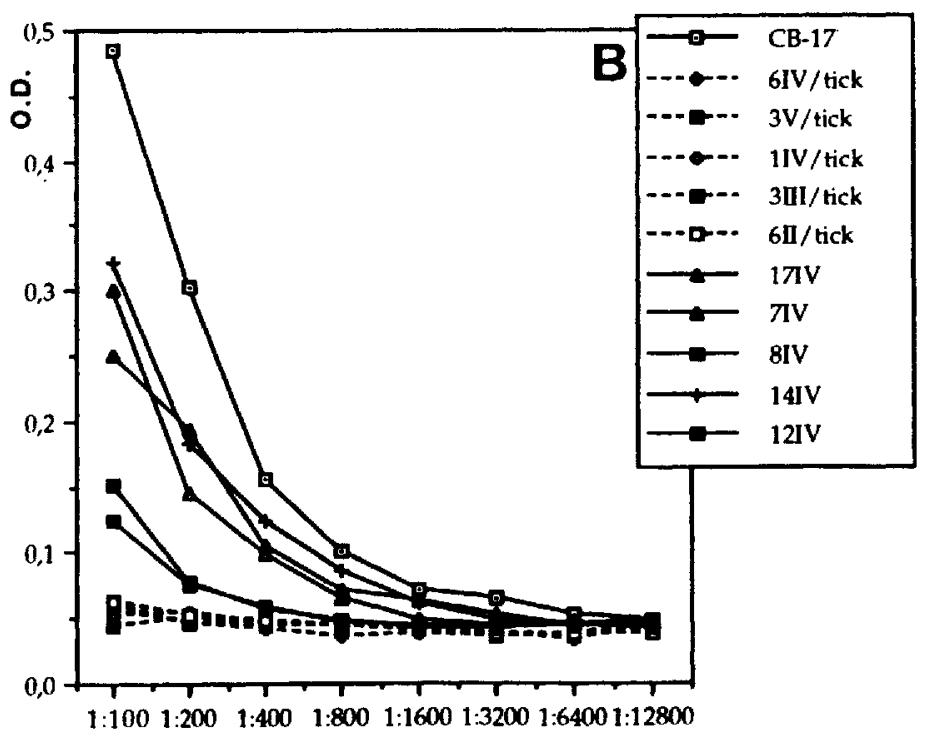

dil

Figure 1. Antibody responses of mice inoculated with $2 \times 10^{7}$ B. burgdorferi (-, mouse 14 and 17), tissue lysates derived from experimentally infected ticks $\left(-, 6 \times 10^{5}\right.$ spirochetes/mouse, mouse 7,8 , and 12), or via experimentally infected ticks (--.-, mouse 1,3 , and 6) by ELISA on whole B. burgdorferi proteins (A) and on recombinant (rec) outer surface protein (Osp) A (B). Sera from mouse strains AKR/N (mouse 1, 7, 8, and 14), DBA/2 (mouse 6, 12, and 17), and C3H/HeJ (mouse 3) were tested. Sera were taken 14 (II), 26 (III), 40 (IV), or 63 (V) days after infection. Pooled immune serum derived from 5 CB 17 mice was used as control $(64 \mu \mathrm{L} / \mathrm{mL}$ specific immunoglobulin). OD, optical density; dil, dilution. 
up

I

II

III

day

7142640

7142640

7142640

$\mathrm{kDa}$

AKR $/ \mathbb{N}$
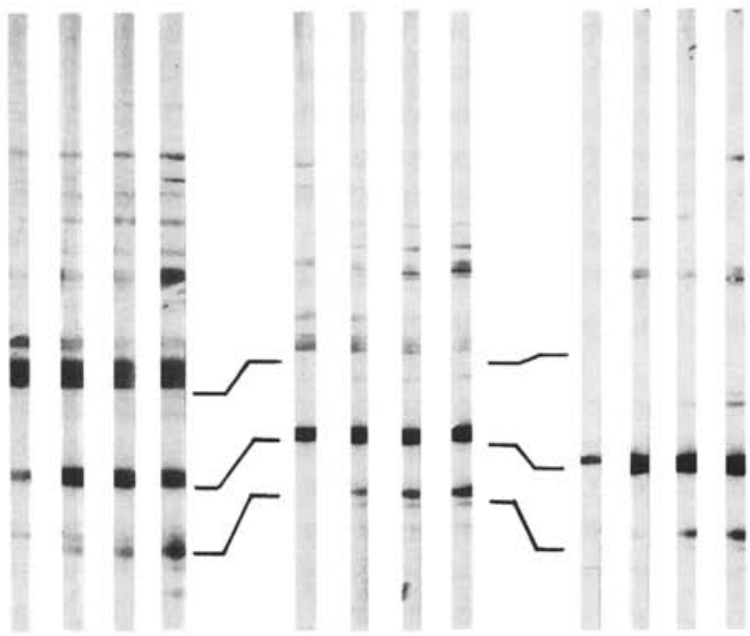

$-96$

$-70$

.44

$-28$

OspB
Ospa

$-18$

$-14$

\section{$\mathrm{C} 3 \mathrm{H} / \mathrm{HeJ}$}
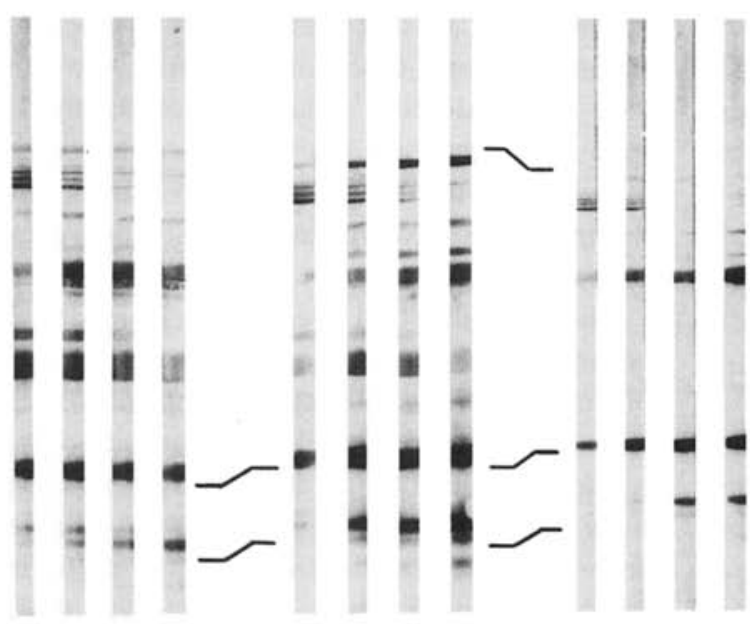

$-96$

$-70$

$-44$

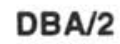

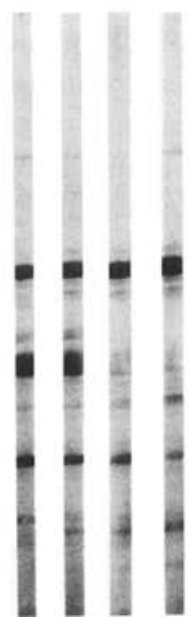

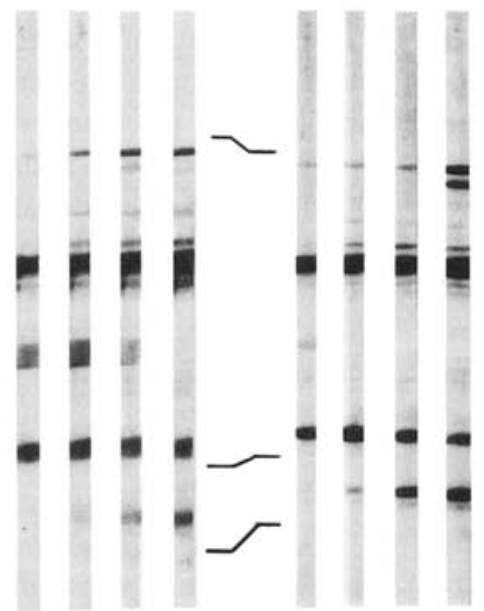

Figure 2. Western blot analysis of sera taken from individual mice of strains $A K R / N, C 3 H / H e J$, and DBA/2 at indicated days after infection. Mice were inoculated with $2 \times 10^{7} B$. burgdorfori spirochetes in BSK II (I), tissue lysates from experimentally infected ticks $(6 \times$ $10^{5}$ /mouse, II), or via experimentally infected ticks (III). Representative data from 1 mouse of each group are shown. Osp, outer surface protein; fla, flagellin.
$-28$

$-18$

$-14$

$-44$

OspB
OspA

18

$(14$

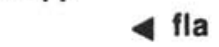

$-28$

4 OspB

4 Ospa 
ticks (group III) contained no or few antibodies to OspA and OspB but had a banding pattern similar to that of the sera from groups I and II. Similar results were obtained with sera taken at days 63,77, and 110 (data not shown).

\section{Discussion}

This investigation showed that the rate of transmission of spirochetes to noninfected tick larvae is much higher in mice infected via tick bites than in those experimentally inoculated by needle injection and that antibodies to OspA and OspB are generated only in response to experimentally but not to tick-delivered organisms.

At first glance, the finding that naturally infected mice in turn infect more ticks than mice experimentally inoculated with cultured spirochetes is surprising, since the number of spirochetes transfered by needle inoculation was at least two to three orders of magnitude higher. This discrepancy may be related to the site of infection, the immune reaction of the host, the number of spirochetes transferred (antigenic load), or the phenotypic changes of the spirochetes within the vector or host or both.

It is possible that skin areas chosen by ticks for blood meals are more heavily infested with spirochetes whereas others are relatively free of the organism. In that case, more naive ticks would become infected when feeding on a recipient that was itself infected by tick bites. At present, detailed information on that point is lacking and requires more intensive studies. It is more likely that the discrepancy of transmission rates observed in naturally versus experimentally infected mice is due to qualitative differences in their immune responses. In fact, sera from all mice infected by any of the three protocols contained similar quantities of spirochetespecific antibodies independent of the greatly differing numbers of spirochetes transferred $\left(<10^{6}\right.$ by tick bites, $6 \times 10^{5}-2$ $\times 10^{7}$ by needle infection); however, antibodies to OspA or OspB were observed only in mice experimentally inoculated by needle injection and not in those infected via tick bites. The finding that the transmission of $B$. burgdorferi organisms to ticks correlated inversely with the presence of antibodies to OspA and OspB in mice therefore suggests that these antibodies are responsible for the elimination of spirochetes and consequently for the reduction in the rate of transmission. This assumption is also supported by previous studies showing that OspA- and OspB-specific antibodies protect SCID $[4,5]$ and $\mathrm{C} 3 \mathrm{H} / \mathrm{HeJ}$ mice $[6,7]$ against infection and development of arthritis after experimental and tick-mediated infection with $B$. burgdorferi, when these antibodies are present at the time of inoculation $[13,14]$.

The finding that the humoral immune response to tick-delivered spirochetes did not include recognition of OspA and OspB is analogous to the situation in patients with Lyme disease, who develop these antibodies, if at all, only during later stages of the infection [8]. It is therefore possible that the immune response of the host is influenced by inoculation route. This hypothesis is substantiated by previous studies demonstrating that experimentally infected dogs [9] and inbred [1, 3, 13] and wild mice (Kurtenbach K. personal communication) express antibodies to OspA and OspB. whereas naturally infected animals do not. The host immune system may indeed be affected by the tick itself, which has been shown before to secrete immunomodulatory constituents during feeding [15]. However, such a mechanism is hard to reconcile with the fact that the suppression seen affects antibodies to OspA and OspB but not to other spirochetal antigens, including flagellin.

It is more likely that the inability of naturally infected mice to produce antibodies to $O \operatorname{spA}$ and $O \operatorname{spB}$ is due to suboptimal doses of spirochetes delivered during the tick's blood meal. This assumption is strongly supported by recent studies showing that mice experimentally inoculated with different numbers of spirochetes $\left(10^{1}-10^{8}\right)$ express similar quantities of antibodies to $B$. burgdorferi but that those with specificities for OspA and $O s p B$ are seen only in animals receiving $>10^{4}$ organisms (unpublished data). These results not only indicate that the number of spirochetes transmitted by ticks during a blood meal is rather low but also suggest that the qualitative differences of humoral immune responses observed are due to differential immunogenicity of individual antigens, such as $\operatorname{OspA}, \operatorname{OspB}$, and flagellin, in the inoculum. Experiments to test this hypothesis are underway.

The possibility that phenotypic changes of the spirochetes occurring in the tick or mouse environment (or both) as demonstrated previously for certain isolates [16] may contribute to the suppression of antibody responses to $O \operatorname{spA}$ and $O \operatorname{spB}$ cannot be formally excluded; however, this seems less likely, since similar phenotypic patterns were obtained with the in vitro-propagated strain ZS7 and its reisolates from either the vector or the murine host. Compared with the first xenodiagnosis at 14 days after infection, the percentage of infected ticks increased from two- to sixfold in all experimental groups of mice at day 100 after infection. These data suggest that spirochetes may survive or multiply in mice even if protective antibodies are generated early during disease. It is possible that the kinetics of appearance of anti-OspA and anti-OspB antibodies and/or their serum level only controls the development of disease, as demonstrated previously [4, 13], but are insufficient to eradicate the spirochetes from the host. Further studies will be directed toward the development of immunization protocols that allow the establishment of sterile immunity against $B$. burgdorferi infection in this animal model.

\section{Acknowledgments}

We thank Olivier Rais and Gabi Nerz for technical assistance, Jean Langhorne for critically reading the manuscript, and Rose Brugger and Gabi Prosch for preparing the manuscript. 


\section{References}

I. Benach JL, Coleman JL, Garcia-Monco JCG, Deponte PC. Biologica activity of Borrelia burgderferi antigens. Ann NY Acad Sci 1988:539:115-25.

2. Schwan TG, Kime KK, Schrumpf ME, Coe JE. Simpson WJ. Antibody response in white-footed mice (Peromyscus leucopus) experimentally infected with the Lyme disease spirochete (Borrelia burgdorferi). Infect Immun 1989:57:3445-51.

3. Schaible UE, Kramer MD, Wallich R, Tran T, Simon MM. Experimental Borrelia burgdorferi infection in inbred mouse strains: antibody response and association of $\mathrm{H}-2$ genes with resistance and susceptibility to development of arthritis. Eur J Immunol 1991;2 l:2397-405.

4. Schaible UE, Kramer MD, Eichmann K, Modolell M, Museteanu C, Simon MM. Monoclonal antibodies specific for the outer surface protein A (OspA) of Borrelia burgdorferi prevent Lyme borreliosis in severe combined immunodeficiency (scid) mice. Proc Natl Acad Sci USA $1990 ; 87: 3768-72$.

5. Simon M, Schaible UE, Kramer MD, et al. Recombinant outer surface protein A from Borrelia burgdorferi induces antibodies protective against spirochetal infection in mice. J Infect Dis 1991;164:123-32.

6. Fikrig E, Barthold SW. Kantor FS. Flawell RA. Protection of mice against the Lyme disease agent by immunizing with recombinant OspA. Science 1991:250:553-6.

7. Fikrig E. Barthold SW, Marcantonio N, Deponte K, Kantor FS, Flavell RA. Roles of OspA, OspB, and flagellin in protective immunity to Lyme borreliosis in laboratory mice. Infect Immun 1992;60:657-61.
8. Steere AC. Lyme disease. N Engl J Med 1989;321:586-96.

9. Greene RT, Walker RL. Nicholson WL, et al. Immunoblot analysis of immunoglobulin $\mathrm{G}$ response to the Lyme disease agent (Borrelia burgdorferi) in experimentally and naturally infected dogs. $\mathrm{J}$ Clin Microbiol 1988;26:648-53.

10. Schaible UE, Kramer MD. Museteanu C. Zimmer G, Mossmann H, Simon MM. The severe combined immunodeficiency (scid) mouse: a laboratory model for the analysis of Lyme arthritis and carditis. J Exp Med 1989;170:1427-32.

11. Gern L, Zhu Z, Aeschlimann A. Development of Borrelia burgdorferi in Ixodes ricinus females during blood feeding. Ann Parasitol Hum Comp 1990;65:89-93.

12. Gern L. Toutoungi LN, Hu CM, Aeschlimann A. Ixodes (Pholeoixodes) hexagonus, an efficient vector of Borrelia burgdorferi in the laboratory. Med Vet Entomol 1991:5:431-5.

13. Schaible UE, Wallich R, Kramer MD, et al. The role of the immune response in Lyme disease: lessons from the mouse model. Curr Comm Cell Mol Biol 1992;6:243-62.

14. Fikrig E, Telford SR III, Barthold SW, Kantor FS, Spielman A, Flavell RA. Elimination of Borrelia burgdorferi from vector ticks feeding on OspA-immunized mice. Proc Natl Acad Sci USA 1990;89:5418-21.

15. Ribeiro JMC. Role of saliva in tick/host interactions. Exp Appl Acarol 1989:7:15-20.

16. Hu CM, Gern L, Aeschlimann A. Changes in protein profile and antigenicity of Borrelia burgdorferi after reintroduction to Ixodes ricinus ticks. Parasite Immunol 1992;14:415-28.

\title{
DNA Fragment Length Polymorphism Analysis of Mycobacterium tuberculosis Isolates by Arbitrarily Primed Polymerase Chain Reaction
}

\author{
Prasit Palittapongarnpim, Sylvia Chomyc, \\ Anne Fanning, and Dennis Kunimoto
}

Department of Medical Microbiology and Infectious Diseases, Provincial Laboratory of Public Health for Northern Alberta; Department of Medicine, University of Alberta. Edmonton. Canada

\begin{abstract}
Strain identification of Mycobacterium tuberculosis would prove whether transmission had occurred between individuals. A method to characterize strains of $M$. tuberculosis has been developed utilizing polymerase chain reaction (PCR). Purified chromosomal DNA of cultured clinical samples of $M$. tuberculosis were subjected to PCR using short (10-12 nucleotide) oligonucleotide primers. PCR products visualized after agarose gel electrophoresis and ethidium bromide staining demonstrated that different strains of $M$. tuberculosis give different banding patterns. This technique was used to confirm the relationship between cases of tuberculosis in several clusters, prove the lack of relationship between 2 isolates with the same antibiotic-resistance pattern, confirm a suspected mislabeling event, and suggest the source of infection in a case of tuberculous meningitis. This method is rapid and simple and does not require radioactive probes.
\end{abstract}

Tuberculosis control in developed countries is based mainly on the interruption of the disease transmission by case finding and treatment. Strategies for case finding gener-

Received 13 July 1992; revised 5 November 1992.

Financial support: Canadian International Development Agency, Alberta Lung Association, and Alberta Heritage Foundation (D.K. is a foundation clinical investigator).

Reprints or correspondence: Dr. D. Y. Kunimoto, Dept. of Medical Microbiology and Infectious Diseases, 1-41 Medical Sciences Bldg., University of Alberta, Edmonton, Alberta, Canada T6G 2H7.

The Journal of Infectious Diseases 1993;167:975-8 (C) 1993 by The University of Chicago. All rights reserved. $0022-1899 / 93 / 6704-0030 \$ 01.00$ ally include case tracing from an index patient with the assumption of airborne spread to individuals in close contact. Direct proof of transmission by strain identification of the organisms is rarely done since all strains of Mycobacterium tuberculosis are serologically homogeneous [1]. Until recently, strain identification could be done only by phage typing. which is time-consuming and technically demanding. Analysis of the resistance profile is of limited value since the number of drugs is finite and an identical resistance profile does not prove that the strains are identical.

Restriction fragment length polymorphism (RFLP) can differentiate strains of $M$. tuberculosis. RFLP could be analyzed after ethidium bromide staining [2] or after Southern 